\title{
Risk factors, contemporary challenges and psychological well-being of the Rohingya refugees in Bangladesh: Policy implications
}

Muhammad Anwar Hossain ${ }^{1}$, Md Nazmul Huda ${ }^{1}$, AKM Ahsan Ullah ${ }^{1}$, and Andre Renzaho $^{1}$

${ }^{1}$ Affiliation not available

September 8, 2021

\section{Hosted file}

1. draft_Proof_hi.pdf available at https://authorea.com/users/431960/articles/535443-riskfactors-contemporary-challenges-and-psychological-well-being-of-the-rohingya-refugeesin-bangladesh-policy-implications 